\title{
Perspective on musculoskeletal modelling and predictive simulations of human movement to assess the neuromechanics of gait
}

Friedl De Groote, Department of Movement Sciences, KU Leuven, Belgium

Antoine Falisse, Department of Bioengineering, Stanford University, USA;

Department of Movement Sciences, KU Leuven, Belgium,

\section{Keywords}

trajectory optimization, optimal control, locomotion

\begin{abstract}
Locomotion results from complex interactions between the central nervous system and the musculoskeletal system with its many degrees of freedom and muscles. Gaining insight into how the properties of each subsystem shape human gait is challenging as experimental methods to manipulate and assess isolated subsystems are limited. Simulations that predict movement patterns based on a mathematical model of the neuro-musculoskeletal system without relying on experimental data can reveal principles of locomotion by elucidating cause-effect relationships. New computational approaches have enabled the use of such predictive simulations with complex neuro-musculoskeletal models. Here, we review recent advances in predictive simulations of human movement and how those simulations have been used to deepen our knowledge about the neuromechanics of gait. In addition, we give a perspective on challenges towards using predictive simulations to gain new fundamental insight into motor control of gait, and to help design personalised treatments in patients with neurological disorders and assistive devices that improve gait performance. Such applications will require more detailed neuro-musculoskeletal models and simulation approaches that take uncertainty into account, tools to efficiently personalize those models, and validation studies to demonstrate the ability of simulations to predict gait in novel circumstances.
\end{abstract}

\section{Introduction}

Notwithstanding decades of research, there remain many open questions about the neuromechanics of human locomotion. Our limited fundamental knowledge hinders the development of novel approaches to improve gait performance in both patients and athletes. A better understanding of the principles underlying human locomotion would benefit the design of exoskeletons that reduce metabolic cost or orthopedic treatments that improve gait performance in patients with neuro-muscular disorders. Locomotion results from complex interactions between the central nervous system, the sensory system and the 
musculoskeletal system with its many degrees of freedom (DoFs) and muscles. Probing the function of each subcomponent is hard, since the invasiveness of available techniques limits direct measurements in humans. In addition, it is hard to experimentally investigate the effect of isolated changes in the neuro-musculoskeletal system on gait performance. Predictive simulations can reveal principles of locomotion by elucidating cause-effect relationships. Such simulations generate novel movements based on a mathematical description, i.e., a model, of the neuro-musculoskeletal system without relying on experimental data (Figure 1). They therefore allow studying the influence of isolated neuromusculoskeletal features by adjusting model parameters. Predictive simulation studies have mostly used conceptual models with few DoFs and without muscles, thereby limiting the questions they could address. Computational roadblocks have long hindered the use of more complex models. However, recent developments in computational approaches have provided powerful tools for simulating movement of complex bodily systems allowing researchers to address long-standing open questions.

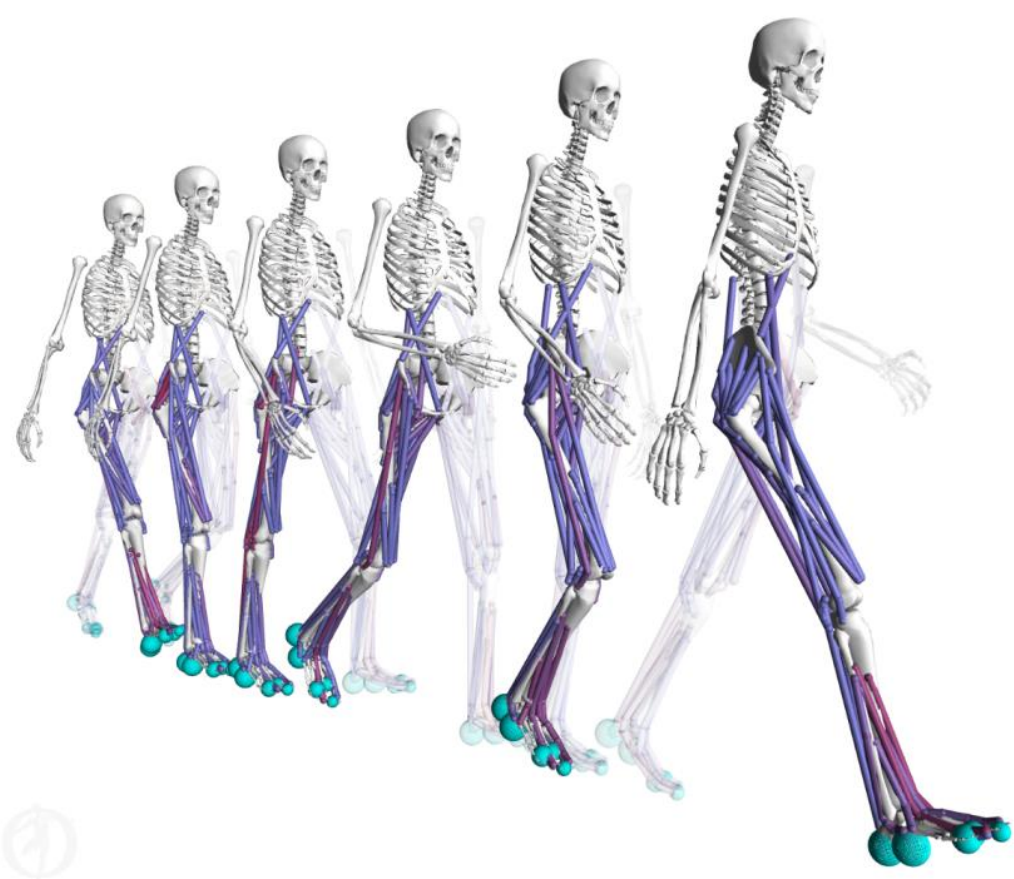

Figure 1: Predictive simulation of human walking. The walking pattern visualised here was generated by trajectory optimization based on a complex OpenSim-based three-dimensional muscle-driven model without relying on experimental data (1)(2). Blue spheres represent contact geometries. Muscles turn red when active.

Conceptual models have advanced our insight into principles of locomotion (3). Simple models of gait have very few parameters and therefore allow exploring the whole parameter space, which makes them amenable to interpretation (4). Such conceptual models demonstrated that energy minimization explains many spatio-temporal gait features, for example the relationship between gait speed and step frequency (5) and the selection of walking and running gaits over other feasible gait patterns (4). Alternatively, 
these models describe the importance of leg compliance to explain the salient dynamics of human gait (6). Conceptual models also revealed principles of gait stability. For example, passive dynamics can stabilize walking in the sagittal plane, whereas lateral stability requires active control (7). When applied to running, conceptual models revealed that less precise control of leg stiffness and angle of attack is required as running speed increases (8) and that local positive muscle force feedback can control leg stiffness (9). However, being simple by nature, conceptual models cannot capture how multiple body segments, a redundant set of muscles and the central nervous system interact to achieve gait economy and stability.

Complex models might elucidate how the neuro-musculoskeletal system realizes gait principles identified through conceptual models. Here, we consider a model complex when it represents multiple lower limb segments and joints driven by a redundant set of muscletendon actuators with dynamics inspired by muscle physiology. First, taking the complex, three-dimensional (3D), musculoskeletal geometry into account is important to understand both pathological gait and athletic performance. For example, patients with weak hip abductors adopt a Trendelenburg gait pattern with compensations in both the frontal and sagittal planes and sprinters have been shown to have smaller Achilles tendon moment arms and longer toes than non-sprinters (10). Second, accurately modelling muscle-tendon actuators is important to understand movement economy. Muscle dynamics are closely linked to muscle energetics with muscle force being produced at lower metabolic rates when a muscle works isometrically close to its optimal length (11). Interactions between muscles and tendons influence muscle efficiency by altering the muscles' operating length and velocity and by allowing for storage and release of energy in the tendons. Third, accurately modelling the musculoskeletal system is crucial to study its interaction with the neural system. The intrinsic mechanical properties of the musculoskeletal system, especially muscles, provide stability against external perturbations, thereby reducing the need for active control (e.g., (12)). Finally, studying how muscles are coordinated by the neural system requires models driven by a redundant set of muscles. Many applications also require such complexity. Using simulations for personalised clinical decision-making requires models that sufficiently describe the musculoskeletal structures and motor control processes that are affected by disease and treatment. For example, children with cerebral palsy often receive orthopedic surgery to correct bony and soft tissue deformities. Integrating predictive simulations in the clinical decision-making process seems promising given the low success rate of such interventions (13) but requires detailed and personalised models. Similarly, using simulations to design exoskeletons that reduce metabolic cost requires models that capture human metabolics, since simply reducing the biological joint torques does not necessarily reduce metabolic cost (14).

Recently, efficient numerical methods and open-source software for predictive simulations based on complex models have become available (2)(15)(16)(17). This availability will enable researchers to address questions about the neuromechanics of gait that require complex models and are hard to study solely based on experiments. Here, we review recent 
advances in predictive simulations of human movement and how those simulations have been used to advance our insight into the neuromechanics of gait. Our review focuses on predictive simulation studies based on complex muscle-driven models. We distinguish predictive simulations, which do not require experimental movement data as input, from tracking simulations, where errors between simulated and experimental kinematics are minimized or constrained. Although we acknowledge that useful insights have been gained from tracking simulations, we believe that the ability to predict de novo movements opens new avenues for research and applications that could not be realized using tracking simulations. We end this perspective with a discussion of open questions and challenges.

\section{Simulation approaches}

Simulations of movement are typically based on the assumption that the central nervous system optimizes performance, e.g., minimizes the metabolic cost of transport. Under this assumption, predictive simulations can be formulated as optimal control problems. We distinguish approaches that solve for (open-loop) muscle controls, often referred to as trajectory optimization, from approaches that solve for control policies (Figure 2). Such control policies describe the dependence of muscle controls on the state of the musculoskeletal system, i.e., feedback control.

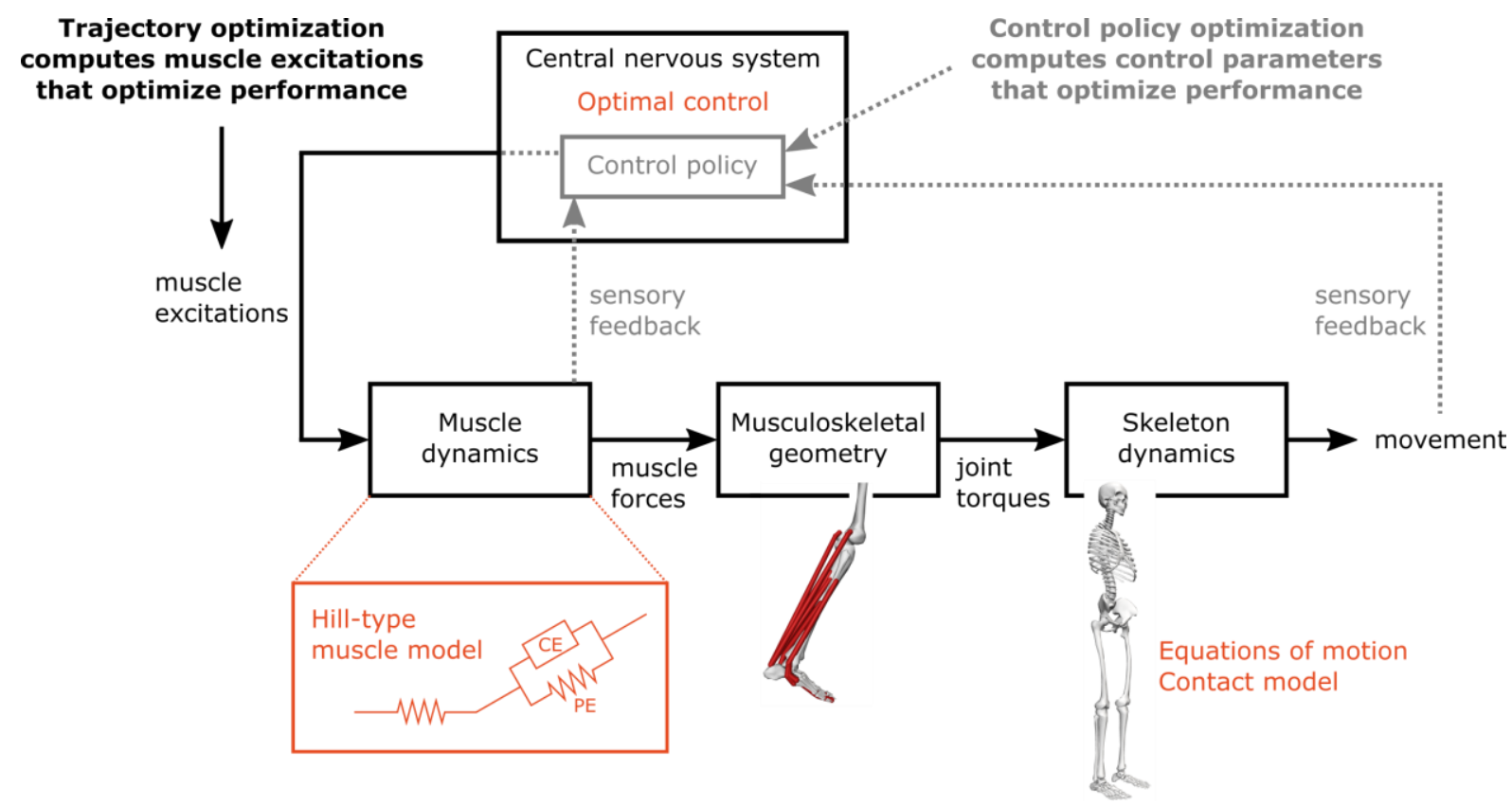

Figure 2: Schematic representation of predictive simulation approaches using trajectory optimization and control policy optimization. Both approaches are based on a model of the musculoskeletal system. Muscle dynamics describe how muscles transform excitations into forces. Commonly, Hill-type muscle models are used. Musculoskeletal geometry determines how these forces are applied to the skeleton system. Skeleton dynamics describe how the skeleton moves under the influence of joint torques and contact forces with the environment. It is commonly assumed that motor control is optimal in some sense, for example by minimizing muscle effort, within the constraints imposed by the musculoskeletal 
system. Trajectory optimization computes open-loop muscle excitations, whereas in control policy optimization the control policy is modeled and control parameters are computed.

Researchers have used trajectory optimization methods with increasing levels of computational efficiency over the last two decades. In 2001, Anderson and Pandy required 10,000 CPU hours to predict a walking cycle that minimized metabolic energy per distance travelled based on a 3D model with 23 DoFs and 54 muscles (18). They used a direct shooting approach combined with a simulated annealing algorithm to solve the underlying optimal control problem. The adoption of direct collocation methods, instead of shooting methods, has helped reduce computational time. In shooting methods, the dynamics are integrated over the time horizon of the simulation based on the current guess of the controls to obtain the states required to evaluate the cost function and constraints. In collocation methods, the discretized states and controls are optimization variables and the integration scheme is expressed by a set of constraints that is solved simultaneously with minimizing the cost function. The resulting large-scale nonlinear programming problems (NLP) are sparse and can be efficiently solved using gradient descent algorithms, e.g., IPOPT (19). Compared to direct shooting, direct collocation reduces the sensitivity of the objective function to the optimization variables by reducing the time horizon of the integration. Hence, collocation methods are especially beneficial to solve problems with unstable dynamics, such as open-loop musculoskeletal dynamics of bipedal locomotion. In 2010, Ackermann and van den Bogert required 35 CPU minutes to predict a walking cycle based on a 2D model with 9 DoFs and 16 muscles (20) and in 2018, Lin et al. required between 13 and 17 CPU hours to predict a walking cycle based on a 3D model with 25 DoFs and 80 muscles (21). Further improvements in computational speed were achieved by combining direct collocation with implicit formulation of the dynamics, which improves the numerical conditioning of the NLP, and algorithmic differentiation (22) (23) (15). In 2019, we required on average 35 CPU minutes to simulate a walking cycle based on a 3D model with 29 DoFs and 92 muscles and shared the computational tools we used on open-source platforms (2). Similar tools have been implemented in OpenSim Moco (16). Moco frees researchers from implementing direct collocation themselves, which makes it easier to use by non-experts. Yet it does not leverage algorithmic differentiation, and might therefore be less computationally efficient. Overall, predictive simulations of walking using trajectory optimization approaches can now be considered rapid and accessible. However, such approaches do not describe gait control policies and are therefore unsuited to describe how the neuro-musculoskeletal system deals with uncertainty, e.g., sensorimotor noise and external perturbations.

Approaches that solve for gait control policies can capture the robustness of the neuromusculoskeletal system against noise, yet are often computationally less efficient than trajectory optimization methods. In 2010, Geyer and Herr proposed a reflex-driven control model of gait (24). They were able to produce stable 2D gait simulations by hand-tuning the control parameters of the model. Since then, their control model has served as a basis for 
optimization approaches that solve for control parameters by optimizing performance. In their 3D model, Wang et al. combined these reflex-based control laws for the 16 muscles driving the sagittal plane lower limb joints with proportional derivative (PD) controllers for the torque actuators driving the remaining 24 DoFs (25). They used Covariance Matrix Adaptation (CMA) to solve for control parameters (26). In 2019, Geijtenbeek et al. published SCONE, an open-source software package to generate predictive simulations by computing control policies using covariance matrix adaptation (17). Alternatively, reinforcement learning has been used to solve for control policies. Since 2017, the NeurIPS "Learn to move" competition series has accelerated the adoption of reinforcement learning techniques to simulate human locomotion based on neuro-musculoskeletal models (for detailed reviews, see (27) and (28)). Overall, approaches that solve for gait control policies were based on simpler neuro-musculoskeletal models than trajectory optimization methods, probably because of computational efficiency. In addition, computational approaches for control policy optimization are typically less suitable for dealing with task constraints, such as periodicity or locomotion speed, than computational approaches for trajectory optimization.

Both trajectory optimization and approaches that solve for gait control policies generated simulations that capture key features of human gait. The realism of simulated movements is typically assessed by comparing simulated and measured joint kinematics, kinetics, ground reaction forces and muscle activities. However, such validation efforts are often qualitative and limited to a single gait pattern. Only few studies went beyond such validation. Song and Geyer solved for gait control policies and validated their simulations against perturbation experiments (29)(30). Their simulations capture the change in muscle activity due to epidural stimulation of the spinal cord, tendon taps, ankle stretch, slip of the stance leg and trip of the swing leg during walking. Dorn et al. validated whether their optimal reflex-based control policies captured key features of level walking as well as inclined and loaded walking (31). We validated that our 3D trajectory optimization simulations captured the relation between gait speed, and respectively gait frequency and metabolic cost of transport (2). Ong et al. performed a similar validation for their reflex-driven 2D simulations (32). However, we feel that further efforts are needed to validate the underlying motor control assumptions. It is clear that control of gait is neither fully open-loop nor fully reflexive. Yet both models have resulted in realistic mechanics and muscle activities, suggesting an important role of musculoskeletal dynamics in shaping human gait.

Relatively few predictive simulation studies have used 3D models with a redundant set of muscles. Yet, given that control of movement is realized through a redundant set of muscles and is coupled between planes, such complexity is required to unveil gait control strategies as well as compensation strategies due to neuro-musculoskeletal deficits . For instance, we observed that we needed to minimize metabolic energy rate squared in addition to muscle activities squared when simulating walking based on a 3D model with a redundant set of muscles to prevent excessive trunk sway in the frontal plane (2), whereas others have 
obtained realistic sagittal plane gait patterns by minimizing only activations squared based on a 2D model in which agonistic mono- and bi-articular actuators were represented by single muscles (20). In addition, we showed that weakening the hip muscles of our 3D model produced walking simulations with excessive hip circumduction, a compensation strategy in the frontal plane (2).

\section{Key insights from predictive simulation studies}

\section{Optimality principles underlying gait control.}

Predictive simulation studies, mostly based on trajectory optimization, have explored optimality principles underlying human gait. Anderson and Pandy showed that minimizing metabolic energy per unit distance travelled of a 3D muscle-driven model produced a walking pattern that closely matched experimental data (18). However, they forced their model to walk with a flexed knee during stance by imposing the joint kinematics at the initial state to match experimental data. Ackermann and van den Bogert compared energylike cost functions, sum of muscle-volume-scaled activations to the power 1-4, and fatiguelike cost functions, sum of activations to the power 2-10 (20). Fatigue-like cost functions predicted larger and more realistic knee flexion during early stance than energy-like cost functions for a 2D muscle-driven model. Based on these results, they suggest that fatigue minimization may be one of the primary optimality principles driving human gait selection. Miller compared the walking patterns predicted by five different metabolic energy models using a 3D muscle-driven model with trapezoidal excitation controls (33). Joint kinematics and ground reaction forces varied with the model used, with some models predicting hip circumduction. It is hard to judge how much the simplified control patterns contributed to differences with respect to human walking. We evaluated the influence of various performance criteria on walking patterns predicted using a 3D muscle-driven model (2). We found that a multi-objective cost function combining metabolic energy, muscle activations and joint accelerations, all terms squared, produced a human-like walking pattern. However, none of the cost functions we tested predicted realistic knee flexion angles and knee extension torques during stance. Our cost function nevertheless captured the walk-to-run transition and clinical gait deficiencies caused by muscle weakness and prosthesis use, suggesting that diverse gaits can emerge from the same optimal control strategy.

Nguyen et al. applied inverse optimal control to identify the cost function underlying a measured walking pattern based on a 2D muscle-driven model (34). They proposed a cost function with terms related to effort, modeled as activations cubed, stability, modeled as the squared horizontal distance between the middle of the base of support and respectively the head and center of mass, and smoothness, modeled as the center of mass jerk squared, and solved for weights that best reproduced experimental data using a bilevel optimization method. Their optimal cost function captures experimental data except for the ankle angle. Note that similar approaches have been applied to 2D torque-driven models (e.g., (35)). 
Optimality principles underlying running have also been studied. For example, Miller et al. compared minimizing the metabolic cost of transport, activations squared and total muscle stress based on a 2D muscle-driven model (36). He found that minimizing activations squared predicted the most realistic joint angles, timing of muscle activity and metabolic cost of transport.

\section{Feasibility of gait control architectures}

Simulations have helped elicit the role of control structures, such as reflexes and central pattern generators, in generating stable and versatile walking patterns. By simulating control architectures, often inspired by experimental observations, researchers have tested their ability to generate human-like walking. Taga proposed a control strategy based on a rhythm generator consisting of seven pairs of neural oscillators with sensory inputs supplemented with joint level impedance controllers to drive a 2D model with ideal muscle actuators, i.e., muscle force proportional to input (37). They fine-tuned the controller parameters through trial and error to generate stable walking. The proposed control architecture was able to generate walking movements at different speeds that were robust against mechanical perturbations. As introduced above, Geyer and Herr proposed a reflexbased control strategy to drive a 2D model with Hill-type muscles (24) and demonstrated that such strategy can generate walking simulations robust against ground disturbances and changes in slope.

Such physiology-inspired control architectures have been combined with performance optimization to test the role of control structures in generating versatile gait. Dzeladini et al. used an optimization approach to demonstrate that a central pattern generator on top of a reflex controller can modulate gait speed based on a 2D muscle-driven model (38). Song and Geyer extended the reflex-based controller of Geyer and Herr to a 3D version that drives 22 muscles actuating eight DoFs and added a supraspinal layer controlling for foot placement, swing leg length and swing leg selection (29). They showed that this control architecture is able to produce diverse steady and transitional locomotion behaviours and therefore suggest that spinal reflexes might be functionally more important than central pattern generators in human locomotion. Song and Geyer evaluated whether the reflex-based control policy that minimized metabolic energy of a 2D model during walking captured observed responses to perturbations (30). The responses of the model were in agreement with experimental observations for local disturbances but were smaller than observed for whole body perturbations, suggesting that the model should be extended with supraspinal control modulating the reflex gains. Wang et al. modeled the supraspinal control layer with a deep neural network that provides input to the reflex-based controller and that was successfully trained to cope with changing terrains (39).

\section{Effect of musculoskeletal properties and neural impairments on gait mechanics and energetics}


Predictive simulations allow assessing how altered neuro-musculoskeletal properties affect gait performance. Such assessment is hard to perform experimentally because - especially with aging or neurological pathologies - multiple physiological changes occur simultaneously. Miller et al. found that maximal sprinting speed predicted by trajectory optimization based on a 2D model driven by Hill-type muscles is more sensitive to the forcevelocity relationship than to the force-length relationship or tendon elasticity (40) (41). Based on a 3D muscle-driven model, Song and Geyer showed that muscular changes, i.e., loss of muscle strength and mass, contribute more to reduced walking economy and speed in older adults than neural changes, i.e., slower neural conduction speed and sensorimotor noise (42). We showed that decreasing the strength of the hip muscles in a 3D muscledriven model produced gaits with excessive hip circumduction, resembling compensated Trendelenburg gait patterns observed in patients with hip muscle weakness, whereas decreasing the strength of the ankle plantarflexors produced calcaneal gaits, which may be observed in children with spastic diplegia who have weak plantarflexors (2). Ong et al. investigated the differential effect of plantarflexor weakness and contraction, often occurring simultaneously in neurological disorders such as cerebral palsy or stroke, on the gait pattern of a 2D muscle-driven model (32). Plantarflexor weakness resulted in slower calcaneal gaits with increased percent time spent in stance, whereas plantarflexor contracture resulted in crouch gait characterized by toe landing and excessive knee and hip flexion. The analyses presented thus far were based on generic models. We also evaluated the differential effects of musculoskeletal and neural impairments on the gait pattern of a child with cerebral palsy using a 3D muscle-driven model with personalised musculoskeletal geometries based on MRI images (43). We found that altered muscle properties, modelled through personalised Hill-type muscle-tendon parameters, rather than spasticity and reduced neuromuscular complexity were the primary contributors to the crouch gait pattern of the child, suggesting that they should be the main treatment targets to restore an upright posture.

\section{Gait patterns and joint loading}

Predictive simulations have been used to study how alterations in the gait pattern might reduce joint loading. Gait retraining is an attractive rehabilitation strategy in joint disorders such as osteoarthritis. Fregly et al. used simulations based on a 3D torque-driven model to design gait modifications that reduced the knee adduction moment while minimizing deviations from the patient's self-selected kinematics (44). They found that slightly increasing leg flexion, decreasing pelvic obliquity and increasing pelvic axial rotation decreased the knee adduction moment. Note that knee adduction moment is a proxy for knee joint loading and that a torque-driven model with simple knee geometry, a pin joint in this case, does not capture the effect of muscle coordination and joint geometry. Miller et al. explored gait modifications that reduced axial knee joint loading based on a 2D muscledriven model (45). They found that reduced gastrocnemius activity, avoidance of knee flexion during stance and smaller strides reduced knee joint loading. Koelewijn and van den 
Bogert found that improving moment symmetry in a 2D muscle-driven simulation of transtibial amputee gait resulted in lower hip and knee contact forces in the intact leg at the cost of increased effort and abnormal kinematics (46).

\section{Design of assistive devices}

The reflex-based model proposed by Geyer and Herr (24) served as inspiration for the design of assistive device controllers. For example, Markowitz et al. used it for controlling an ankle prosthesis and showed that it resulted in a physiological adaptation of ankle work in response to ground slope variation (47). Further, Wu et al. used it for controlling hip and knee torques delivered by an assistive lower-limb exoskeleton, and demonstrated its ability to produce near-physiological walking kinematics at near-normative speeds for subjects with spinal cord injury (48).

Alternatively, simulations have been used to explore the effect of prosthesis design on the gait pattern. Handford and Srinivasan used trajectory optimization based on a 2D muscledriven model to explore the effect of the control strategy on the kinematics and energetics of walking with a transtibial prosthesis (49). They found that both too little and too much prosthesis work increased the metabolic energy rate, and that metabolic energy rate could be reduced when allowing asymmetric walking patterns. Our simulations using trajectory optimization based on a 3D muscle-driven model captured the ankle plantarflexion torque patterns due to passive transtibial prosthetic use (2).

\section{Computer graphics applications}

Muscle-driven models have been adopted in computer graphics due to the increased realism of gait patterns predicted with muscle- versus torque-driven models. Mordatch et al. used trajectory optimization to simulate human gaits and other movements based on a 3D muscle-driven model (50). They minimized dynamic inconsistencies rather than imposing dynamics, reflecting their focus on generating visually plausible movement patterns in a computationally efficient way rather than studying the neuromechanics of human gait. Their simulation produced realistic simulations of steady-state gaits in Earth's and reduced gravity, gait initiation and incline walking.

\section{Challenges and future perspectives}

Predictive simulations have begun to provide valuable insights into the neuromechanics of gait. Recent computational advances will allow us to further increase the realism of the associated neuro-musculoskeletal models, thereby bridging the gap between how we currently conceptualize principles underlying human locomotion and how they are physiologically realized in the neuro-musculoskeletal system. In addition, the use of models that better reflect an individual's neuro-musculoskeletal properties will advance our 
understanding of inter-subject differences in gait patterns. Here, we discuss challenges and future perspectives to increase the accuracy of predictive simulations.

\section{Motor control models}

Translating available knowledge on motor control into models will be important to improve the realism of simulations. By solving for muscle controls that optimize performance, trajectory optimization makes abstraction of underlying control structures contained in the brain and spinal cord and therefore does not give any insight into how the neural system generates muscle controls. This is an important barrier to study how neurological disorders that affect specific regions in the brain or spinal cord impede gait performance. Approaches that predict human walking by computing optimal control policies have mainly relied on reflex-based controllers. Reflex pathways implemented in popular models are simplifications of the reflex system and only part of the muscle input can likely be attributed to reflexes (51). Reflex models have been extended by central pattern generators (38) and supraspinal inputs (29), yet none of these simple control models captures the complexity of human motor control. Important sources of sensory information, such as cutaneous receptors and vision, are often not considered and the process of sensory integration is not explicitly modeled. Developing more accurate motor control models is not straightforward as we lack a comprehensive framework and quantitative data, e.g., on the relative contribution of supraspinal and reflex pathways. We might take inspiration from motor control models that have been used to simulate animal locomotion (e.g., (52)).

Translating clinical definitions of neurological symptoms into mathematical models is important to study how these symptoms affect locomotion. For example, spasticity is a common symptom in neurological disorders that is defined as a velocity-dependent increase in tonic stretch reflexes resulting from hyperexcitability of the stretch reflex (53). This definition has inspired modellers to describe muscle activity due to hyperactive stretch reflexes as feedback from fiber velocity but such models failed to capture key features of the response to passive stretch in individuals with spasticity. Instead, we took inspiration from in vivo muscle stretch experiments in healthy animals (54) to develop more accurate models of spasticity by describing muscle activity due to hyperactive stretch reflexes by feedback from history dependent muscle force (55)(56). Our simulations inspired new experimental studies that confirmed the movement history-dependence of the response to passive stretch in individuals with spastic cerebral palsy (57). Yet studying how spasticity affects whole body movements will require embedding those reflex models into more complete motor control models that capture task-dependent supraspinal modulation of reflexes.

\section{Optimality principles}

There is a need for experiments designed to test potential trade-offs between multiple criteria to advance our understanding of the optimality criteria underlying human 
movement. Both experiments and simulations suggest that energy minimization shapes human gait (58)(4). While it is likely that other criteria also contribute, it remains challenging to identify them. The search space is vast, i.e., infinite combinations of potential criteria could be tested, and redundant, i.e., different criteria might result in very similar simulated walking patterns. An additional problem is that it is hard to distinguish the effect of optimal control assumptions and musculoskeletal modelling errors on the predicted movement patterns.

While trade-offs between criteria might be subtle for steady-state level-ground walking, they might be more pronounced in other conditions (59). Predictive simulations could help identify experimental conditions that would allow distinguishing between optimality criteria. For example, simulations could be used to explore the mechanical constraints (e.g., limited joint range of motion or foot-ground contact locations, slopes or stairs, added segment mass or volume) that lead to the largest predicted difference in kinematics when prioritizing minimizing metabolic cost versus muscle activity squared, which might be a measure of fatigue.

Alternatively, inverse optimal control is a useful tool to identify optimality criteria underlying measured movements. Application of inverse optimal control has, nevertheless, been limited to simple 2D models (35) (34) likely due to the high computational cost of performing bilevel optimization. In addition, inverse optimal control can only be expected to be successful when the experimental input data contains sufficient information to distinguish different criteria.

\section{Robust optimal control}

Todorov and Jordan demonstrated that it was important to account for uncertainty when optimizing task performance to realistically reproduce upper limb movements (60) but uncertainty, e.g., due to sensorimotor noise, is often neglected when simulating gait. When using shooting methods to compute control policies, accounting for uncertainty is computationally straightforward because sensorimotor and external noise can be added when evaluating the dynamics. Yet the high computational cost of current computational approaches for control policy optimization might be a barrier to applying stochastic optimal control to complex models. Since robustness against uncertainty requires feedback control, trajectory optimization is by design unsuited for studying stochastic optimal control. Future methodological developments should therefore aim at leveraging the recent computational advances that improved the efficiency of trajectory optimization to stochastic optimal control. For example, Koelewijn and van den Bogert accounted for control noise when simulating walking based on a torque-driven sagittal plane model (61). They computed open loop controls and gains of reflexive joint controllers by optimizing performance over a set of predefined noise trajectories using direct collocation and gradient-based optimization. Increased control noise resulted in larger foot clearance. We are currently exploring whether the approach suggested by Houska et al. for mechatronic control (62) is applicable 
to simulate human gait. The main idea is to approximate the stochastic state by its mean and covariance, and to approximate the dynamics of the state covariance using the continuous Lyaponuv equation. The resulting approximate deterministic optimal control problem can then be solved with direct collocation. However, it remains to be seen whether computational efficiency can be improved using such approaches given the large size of the resulting optimal control problems.

\section{Musculoskeletal model complexity}

Recent computational advances enable the integration of increasingly complex models of the musculoskeletal system in predictive simulations. Common assumptions in gait simulation studies are to model the feet and the upper body as single rigid segments. Inverse approaches that aim to study joint torques or muscle coordination underlying a measured movement might be less sensitive to such assumptions than predictive simulations. For example, modelling the foot as a two-segment versus single segment system has little influence on the knee torque estimated from motion capture data, i.e., marker trajectories and ground reaction forces, whereas in recent work we found that it has a large effect on the knee torque in predictive simulations (unpublished). Model complexity might have important implications for testing optimality principles underlying human gait as well. For example, Song and Geyer found that adaptive feet increased the energetic cost in simulations of walking (63).

Predictive simulations of whole body movements typically rely on phenomenological Hilltype muscle models that might not accurately capture muscle mechanics and energetics. We think that computational speed is an important limitation to the adoption of more complex and realistic models of muscle dynamics. For example, van Soest and Lemaire needed 10,000 times more CPU time to simulate vertical jumping based on a 2D model driven by six muscles when using a Huxley-type cross bridge model instead of a Hill-type muscle model (64). In addition, the unavailability of model parameters for the lower limb muscles as well as approaches to scale them to individual subjects might hinder the adoption of alternative models of muscle dynamics.

Insight into how foot-ground contact models influence predicted walking patterns is limited. Elastic foundation and Hunt-Crossley models are popular to describe contacts. Yet they might not accurately capture contact geometries, potentially affecting the predicted movements. Avoidance of inter-limb collisions has also been shown to shape human gaits (65), but inter-limb contacts are typically not modeled. In recent work, we prevented interlimb collisions by imposing minimum distances between segment origins (2). Contacts are inherently discontinuous and therefore unsuited for gradient-based optimization. To overcome this issue, researchers have either prescribed the sequence of foot-ground contacts, which is undesirable when the contact sequence is unknown a priori, or used smooth approximations of discontinuous models. Alternative contact descriptions have also 
been proposed. For example, Mordatch et al. used a contact model that does not impose the dependence of contact forces on deformation (50).

\section{Between subject variation/personalisation}

Neuro-musculoskeletal models typically represent an average human rather than a specific individual. Methods for image-based modelling of musculoskeletal geometry, i.e., joint definitions and muscle paths, are available (e.g. (66)) but the long time required to build image-based models has limited their use. Similarly, medical imaging can provide information about muscle-tendon properties. However, relating image-based measures and Hill-type model parameters is not always straightforward given the simplified representation of the muscle as a single fibre in series with the tendon in such models. Alternatively, model parameters can be identified based on experimental EMG-torque relationships (e.g., (67) (68)). This approach has been successfully applied to estimate properties of the ankle and knee actuators but the inability to collect surface EMG from deep muscles limits its use for the hip actuators. Similarly, dynamic ultrasound-based measures of fibre lengths can be used for parameter identification but such measures are typically limited to very few muscles (69).

Methods for personalised modelling of motor control are scarce. Little is known about how optimality principles underlying gait differ between subjects or within subjects depending on experience and mood. Liu et al. used inverse optimal control to find the optimality criterion underlying 3D torque-driven simulations that best represented an observed movement style (70). Alternatively, muscle synergies derived from EMG signals measured during walking have been used for personalised modelling of muscle coordination in stroke (71). The disadvantage of this approach is that experimental data from the movement being studied is needed as input and hence the simulations can no longer be considered predictive.

Available methods for model personalisation have in common that they require extensive data collection. Estimation of model parameters based on limited input data is susceptible to overfitting. Predictive simulations could be used to explore the sensitivity of predicted walking patterns to model parameters, thereby enabling targeted selection of model parameters to be personalised and experimental data to be collected.

\section{Personalised treatment selection}

Predictive simulations are an attractive tool for personalised treatment selection. Predictive simulations would allow us to test different treatment options in advance of the intervention and to select the treatment option that has the highest potential to improve gait performance. We currently focus on using predictive simulations to predict the effect of single event multi-level surgery in children with cerebral palsy, where treatment outcome is currently unpredictable and variable (72). Yet it is unclear what level of model complexity and personalisation is needed to distinguish successful from unsuccessful interventions. 
Similarly, predictive simulations could accelerate the design of devices aiming to improve gait performance such as running shoes, insoles, prostheses and exoskeletons but it has yet to be convincingly demonstrated that they can predict the effect of such devices on the gait pattern.

\section{Adaptation, learning and experience}

The ability to simulate how experience shapes gait mechanics and energetics will be important if we want to predict the effect of gait training or adaptation to devices such as exoskeletons. For example, Sellinger et al. showed that humans change their gait pattern when wearing an exoskeleton such that the energetic cost is minimized and that this process requires exploration away from their non-assisted gait pattern (58). But even nongait-specific training might have an influence on gait. Sawers et al. demonstrated that muscle coordination during walking was different in professionally trained ballet dancers as compared to healthy controls (73). Reinforcement learning might offer a framework to translate experimental observations on adaptation, learning, and experience into simulations.

\section{Conclusion}

We expect the use of predictive simulations for investigating the neuromechanics of human gait to grow in the coming years thanks to recent developments in computational approaches and the availability of open-source software packages. Yet challenges remain to further improve the realism of predictive simulations and to validate the simulated outcomes. Addressing these challenges will be important to answer both fundamental questions about the neuromechanics of gait and to use predictive simulations for the design of interventions that improve gait performance.

\section{Data accessibility}

This article has no additional data.

\section{Competing interests}

We declare we have no competing interests.

\section{Funding}

This study was funded by KU Leuven Internal Funds C24M/19/064. 


\section{References}

1. Seth A, Hicks JL, Uchida TK, Habib A, Dembia CL, Dunne JJ, et al. OpenSim:

Simulating musculoskeletal dynamics and neuromuscular control to study human and animal movement. Schneidman D, editor. PLOS Comput Biol. 2018 Jul 26;14(7):e1006223.

2. Falisse A, Serrancolí G, Dembia CL, Gillis J, Jonkers I, De Groote F. Rapid predictive simulations with complex musculoskeletal models suggest that diverse healthy and pathological human gaits can emerge from similar control strategies. J R Soc Interface. 2019 Aug;16(157):20190402.

3. Kuo AD, Donelan JM. Dynamic Principles of Gait and Their Clinical Implications. Phys Ther. 2010 Feb 1;90(2):157-74.

4. Srinivasan M, Ruina A. Computer optimization of a minimal biped model discovers walking and running. Nature. 2006 Jan;439(7072):72-5.

5. Bertram JEA, Ruina A. Multiple Walking Speed-frequency Relations are Predicted by Constrained Optimization. J Theor Biol. 2001 Apr;209(4):445-53.

6. Geyer H, Seyfarth A, Blickhan R. Compliant leg behaviour explains basic dynamics of walking and running. Proc R Soc B Biol Sci. 2006 Nov 22;273(1603):2861-7.

7. Bauby $C E$, Kuo AD. Active control of lateral balance in human walking. J Biomech. 2000 Nov;33(11):1433-40.

8. Seyfarth A, Geyer H, Günther M, Blickhan R. A movement criterion for running. J Biomech. 2002 May;35(5):649-55.

9. Geyer H, Seyfarth A, Blickhan R. Positive force feedback in bouncing gaits? Proc R Soc Lond B Biol Sci. 2003 Oct 22;270(1529):2173-83.

10. Lee SSM, Piazza SJ. Built for speed: musculoskeletal structure and sprinting ability. J Exp Biol. 2009 Nov 15;212(22):3700-7.

11. Hill AV. The heat of shortening and the dynamic constants of muscle. Proc $R$ Soc Lond Ser B - Biol Sci. 1938 Oct 10;126(843):136-95.

12. De Groote F, Allen JL, Ting LH. Contribution of muscle short-range stiffness to initial changes in joint kinetics and kinematics during perturbations to standing balance: A simulation study. J Biomech. 2017 Apr;55:71-7.

13. Filho MC de M, Yoshida R, Carvalho W da S, Stein HE, Novo NF. Are the recommendations from three-dimensional gait analysis associated with better postoperative outcomes in patients with cerebral palsy? Gait Posture. 2008 Aug;28(2):316-22.

14. Jackson RW, Collins SH. An experimental comparison of the relative benefits of work and torque assistance in ankle exoskeletons. J Appl Physiol. 2015 Sep 1;119(5):541-57.

15. Falisse A, Serrancolí G, Dembia CL, Gillis J, De Groote F. Algorithmic differentiation improves the computational efficiency of OpenSim-based trajectory optimization of human movement. Srinivasan M, editor. PLOS ONE. 2019 Oct 17;14(10):e0217730.

16. Dembia CL, Bianco NA, Falisse A, Hicks JL, Delp SL. OpenSim Moco: Musculoskeletal optimal control [Internet]. Bioengineering; 2019 Nov [cited 2020 Sep 27]. Available from: http://biorxiv.org/lookup/doi/10.1101/839381

17. Geijtenbeek T. SCONE: Open Source Software for Predictive Simulation of Biological Motion. J Open Source Softw. 2019 Jun 14;4(38):1421.

18. Anderson FC, Pandy MG. Dynamic Optimization of Human Walking. J Biomech Eng. 2001 Oct 1;123(5):381-90.

19. Wächter A, Biegler LT. On the implementation of an interior-point filter line-search algorithm for large-scale nonlinear programming. Math Program. 2006 Mar;106(1):2557.

20. Ackermann M, van den Bogert AJ. Optimality principles for model-based prediction of human gait. J Biomech. 2010 Apr;43(6):1055-60.

21. Lin Y-C, Walter JP, Pandy MG. Predictive Simulations of Neuromuscular Coordination and Joint-Contact Loading in Human Gait. Ann Biomed Eng. 2018 Aug;46(8):1216-27. 
22. van den Bogert AJ, Blana D, Heinrich D. Implicit methods for efficient musculoskeletal simulation and optimal control. Procedia IUTAM. 2011;2:297-316.

23. De Groote F, Kinney AL, Rao AV, Fregly BJ. Evaluation of Direct Collocation Optimal Control Problem Formulations for Solving the Muscle Redundancy Problem. Ann Biomed Eng. 2016 Oct;44(10):2922-36.

24. Geyer H, Herr H. A Muscle-Reflex Model That Encodes Principles of Legged Mechanics Produces Human Walking Dynamics and Muscle Activities. IEEE Trans Neural Syst Rehabil Eng. 2010 Jun;18(3):263-73.

25. Wang JM, Hamner SR, Delp SL, Koltun V. Optimizing locomotion controllers using biologically-based actuators and objectives. ACM Trans Graph. 2012 Aug 5;31(4):1-11.

26. Hansen N. The CMA Evolution Strategy: A Comparing Review. In: Lozano JA, Larrañaga P, Inza I, Bengoetxea E, editors. Towards a New Evolutionary Computation. Berlin, Heidelberg: Springer Berlin Heidelberg; 2006. p. 75-102. (Studies in Fuzziness and Soft Computing; vol. 192).

27. Kidziński Ł, Mohanty SP, Ong CF, Huang Z, Zhou S, Pechenko A, et al. Learning to Run Challenge Solutions: Adapting Reinforcement Learning Methods for

Neuromusculoskeletal Environments. In: Escalera S, Weimer M, editors. The NIPS '17 Competition: Building Intelligent Systems. Cham: Springer International Publishing; 2018. p. 121-53.

28. Song S, Kidziński Ł, Peng XB, Ong C, Hicks J, Levine S, et al. Deep reinforcement learning for modeling human locomotion control in neuromechanical simulation [Internet]. Bioengineering; 2020 Aug [cited 2020 Sep 27]. Available from: http://biorxiv.org/lookup/doi/10.1101/2020.08.11.246801

29. Song S, Geyer H. A neural circuitry that emphasizes spinal feedback generates diverse behaviours of human locomotion: A spinal feedback circuitry generating human locomotion behaviors. J Physiol. 2015 Aug 15;593(16):3493-511.

30. Song S, Geyer H. Evaluation of a Neuromechanical Walking Control Model Using Disturbance Experiments. Front Comput Neurosci. 2017 Mar 14;11.

31. Dorn TW, Wang JM, Hicks JL, Delp SL. Predictive Simulation Generates Human Adaptations during Loaded and Inclined Walking. Zadpoor AA, editor. PLOS ONE. 2015 Apr 1;10(4):e0121407.

32. Ong CF, Geijtenbeek T, Hicks JL, Delp SL. Predicting gait adaptations due to ankle plantarflexor muscle weakness and contracture using physics-based musculoskeletal simulations. Srinivasan M, editor. PLOS Comput Biol. 2019 Oct 7;15(10):e1006993.

33. Miller $\mathrm{RH}$. A comparison of muscle energy models for simulating human walking in three dimensions. J Biomech. 2014 Apr;47(6):1373-81.

34. Nguyen VQ, Johnson RT, Sup FC, Umberger BR. Bilevel Optimization for Cost Function Determination in Dynamic Simulation of Human Gait. IEEE Trans Neural Syst Rehabil Eng. 2019 Jul;27(7):1426-35.

35. Mombaur K, Truong A, Laumond J-P. From human to humanoid locomotion-an inverse optimal control approach. Auton Robots. 2010 Apr;28(3):369-83.

36. Miller $\mathrm{RH}$, Umberger BR, Hamill J, Caldwell GE. Evaluation of the minimum energy hypothesis and other potential optimality criteria for human running. Proc R Soc B Biol Sci. 2012 Apr 22;279(1733):1498-505.

37. Taga G. A model of the neuro-musculo-skeletal system for human locomotion: II. Realtime adaptability under various constraints. Biol Cybern. 1995 Jul;73(2):113-21.

38. Dzeladini F, van den Kieboom J, ljspeert A. The contribution of a central pattern generator in a reflex-based neuromuscular model. Front Hum Neurosci. 2014 Jun 26;8.

39. Wang J, Qin W, Sun L. Terrain Adaptive Walking of Biped Neuromuscular Virtual Human Using Deep Reinforcement Learning. IEEE Access. 2019;7:92465-75.

40. Miller RH, Umberger BR, Caldwell GE. Limitations to maximum sprinting speed imposed by muscle mechanical properties. J Biomech. 2012 Apr;45(6):1092-7.

41. Miller RH, Umberger BR, Caldwell GE. Sensitivity of maximum sprinting speed to characteristic parameters of the muscle force-velocity relationship. J Biomech. 2012 May;45(8):1406-13. 
42. Song S, Geyer H. Predictive neuromechanical simulations indicate why walking performance declines with ageing: Computer simulations of elderly gait. J Physiol. 2018 Apr 1;596(7):1199-210.

43. Falisse A, Pitto L, Kainz H, Hoang H, Wesseling M, Van Rossom S, et al. Physics-Based Simulations to Predict the Differential Effects of Motor Control and Musculoskeletal Deficits on Gait Dysfunction in Cerebral Palsy: A Retrospective Case Study. Front Hum Neurosci. 2020 Feb 18;14:40.

44. Fregly BJ, Reinbolt JA, Rooney KL, Mitchell KH, Chmielewski TL. Design of patientspecific gait modifications for knee osteoarthritis rehabilitation. IEEE Trans Biomed Eng. 2007 Sep;54(9):1687-95.

45. Miller RH, Brandon SCE, Deluzio KJ. Predicting Sagittal Plane Biomechanics That Minimize the Axial Knee Joint Contact Force During Walking. J Biomech Eng. 2013 Jan 1;135(1):011007.

46. Koelewijn $A D$, van den Bogert AJ. Joint contact forces can be reduced by improving joint moment symmetry in below-knee amputee gait simulations. Gait Posture. 2016 Sep;49:219-25.

47. Markowitz J, Krishnaswamy P, Eilenberg MF, Endo K, Barnhart C, Herr H. Speed adaptation in a powered transtibial prosthesis controlled with a neuromuscular model. Philos Trans R Soc B Biol Sci. 2011 May 27;366(1570):1621-31.

48. Wu AR, Dzeladini F, Brug TJH, Tamburella F, Tagliamonte NL, van Asseldonk EHF, et al. An Adaptive Neuromuscular Controller for Assistive Lower-Limb Exoskeletons: A Preliminary Study on Subjects with Spinal Cord Injury. Front Neurorobotics. 2017 Jun 20;11:30.

49. Handford ML, Srinivasan M. Robotic lower limb prosthesis design through simultaneous computer optimizations of human and prosthesis costs. Sci Rep. 2016 Apr;6(1):19983.

50. Mordatch I, Todorov E, Popović Z. Discovery of complex behaviors through contactinvariant optimization. ACM Trans Graph. 2012 Aug 5;31(4):1-8.

51. Sinkjaer T, Andersen JB, Ladouceur M, Christensen LOD, Nielsen JB. Major role for sensory feedback in soleus EMG activity in the stance phase of walking in man. $J$ Physiol. 2000 Mar;523(3):817-27.

52. Ekeberg Ö, Pearson K. Computer Simulation of Stepping in the Hind Legs of the Cat: An Examination of Mechanisms Regulating the Stance-to-Swing Transition. J Neurophysiol. 2005 Dec;94(6):4256-68.

53. Lance JW. The control of muscle tone, reflexes, and movement: Robert Wartenbeg Lecture. Neurology. 1980 Dec 1;30(12):1303-1303.

54. Blum KP, Lamotte D'Incamps B, Zytnicki D, Ting LH. Force encoding in muscle spindles during stretch of passive muscle. Ayers J, editor. PLOS Comput Biol. 2017 Sep 25;13(9):e1005767.

55. Falisse A, Bar-On L, Desloovere K, Jonkers I, De Groote F. A spasticity model based on feedback from muscle force explains muscle activity during passive stretches and gait in children with cerebral palsy. Di Giminiani R, editor. PLOS ONE. 2018 Dec 7;13(12):e0208811.

56. De Groote F, Blum KP, Horslen BC, Ting LH. Interaction between muscle tone, shortrange stiffness and increased sensory feedback gains explains key kinematic features of the pendulum test in spastic cerebral palsy: A simulation study. Ivanenko YP, editor. PLOS ONE. 2018 Oct 18;13(10):e0205763.

57. Willaert J, Desloovere K, Van Campenhout A, Ting LH, De Groote F. Movement History Influences Pendulum Test Kinematics in Children With Spastic Cerebral Palsy. Front Bioeng Biotechnol. 2020 Aug 7;8:920.

58. Selinger JC, O'Connor SM, Wong JD, Donelan JM. Humans Can Continuously Optimize Energetic Cost during Walking. Curr Biol. 2015 Sep;25(18):2452-6.

59. McDonald KA, Cusumano JP, Peeling P, Rubenson J. Multi-objective control in human walking: insight gained through simultaneous degradation of energetic and motor regulation systems. J R Soc Interface. 2019 Sep;16(158):20190227.

60. Todorov E, Jordan MI. Optimal feedback control as a theory of motor coordination. Nat 
Neurosci. 2002 Nov;5(11):1226-35.

61. Koelewijn AD, van den Bogert AJ. A solution method for predictive simulations in a stochastic environment. J Biomech. 2020 May;104:109759.

62. Houska B, Diehl M. Robustness and stability optimization of power generating kite systems in a periodic pumping mode. In: 2010 IEEE International Conference on Control Applications. 2010. p. 2172-7.

63. Song S, Geyer H. The energetic cost of adaptive feet in walking. In: 2011 IEEE International Conference on Robotics and Biomimetics. 2011. p. 1597-602.

64. van Soest AJ "Knoek," Casius LJR, Lemaire KK. Huxley-type cross-bridge models in largeish-scale musculoskeletal models; an evaluation of computational cost. J Biomech. 2019 Jan;83:43-8.

65. Afschrift M, Pitto L, Aerts W, van Deursen R, Jonkers I, De Groote F. Modulation of gluteus medius activity reflects the potential of the muscle to meet the mechanical demands during perturbed walking. Sci Rep. 2018 Dec;8(1):11675.

66. Arnold AS, Salinas S, Hakawa DJ, Delp SL. Accuracy of Muscle Moment Arms Estimated from MRI-Based Musculoskeletal Models of the Lower Extremity. Comput Aided Surg. 2000 Jan;5(2):108-19.

67. Lloyd DG, Besier TF. An EMG-driven musculoskeletal model to estimate muscle forces and knee joint moments in vivo. J Biomech. 2003 Jun;36(6):765-76.

68. Falisse A, Van Rossom S, Jonkers I, De Groote F. EMG-Driven Optimal Estimation of Subject-SPECIFIC Hill Model Muscle-Tendon Parameters of the Knee Joint Actuators. IEEE Trans Biomed Eng. 2017 Sep;64(9):2253-62.

69. Delabastita T, Afschrift M, Vanwanseele B, De Groote F. Ultrasound-Based Optimal Parameter Estimation Improves Assessment of Calf Muscle-Tendon Interaction During Walking. Ann Biomed Eng. 2020 Feb;48(2):722-33.

70. Liu CK, Hertzmann A, Popović Z. Learning physics-based motion style with nonlinear inverse optimization. ACM Trans Graph. 2005 Jul;24(3):1071-81.

71. Meyer AJ, Eskinazi I, Jackson JN, Rao AV, Patten C, Fregly BJ. Muscle Synergies Facilitate Computational Prediction of Subject-Specific Walking Motions. Front Bioeng Biotechnol. 2016 Oct 13;4.

72. Schwartz MH. O 046 - A flexible omnibus matching algorithm (FOMA) to support treatment decisions for children with cerebral palsy. Gait Posture. 2018 Sep;65:93-4.

73. Sawers A, Allen JL, Ting LH. Long-term training modifies the modular structure and organization of walking balance control. J Neurophysiol. 2015 Dec 1;114(6):3359-73. 